\title{
Using DAE Solvers to Examine Local Identifiability for Linear and Nonlinear Systems
}

\author{
Markus Gerdin \\ Division of Automatic Control \\ Department of Electrical Engineering \\ Linköpings universitet, SE-581 83 Linköping, Sweden \\ WWW: http://Www. control.isy.liu.se \\ E-mail: gerdin@isy.liu.se
}

5th December 2005

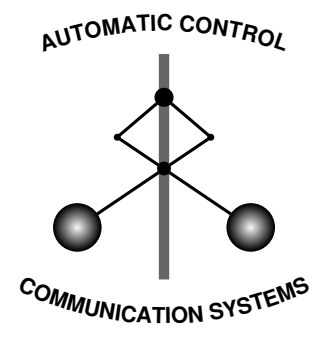

LINKÖPING

Report no.: LiTH-ISY-R-2712

Submitted to 14th IFAC Symposium on System Identification, SYSID-2006

Technical reports from the Control \& Communication group in Linköping are available at http://www.control.isy.liu.se/publications. 


\begin{abstract}
If a model structure is not identifiable, then it is not possible to uniquely identify its parameters from measured data. This contribution describes how solvers for differential-algebraic equations (DAE) can be used to examine if a model structure is locally identifiable. The procedure can be applied to both linear and nonlinear systems. If a model structure is not identifiable, it is also possible to examine which functions of the parameters that are locally identifiable.
\end{abstract}

Keywords: Identifiability, Nonlinear systems, Modelling, Identification, Descriptor systems 


\title{
USING DAE SOLVERS TO EXAMINE LOCAL IDENTIFIABILITY FOR LINEAR AND NONLINEAR SYSTEMS ${ }^{1}$
}

\author{
Markus Gerdin \\ gerdin@isy.liu.se \\ Division of Automatic Control, Department of Electrical \\ Engineering, Linköping University, SE-581 83 Linköping, \\ Sweden
}

\begin{abstract}
If a model structure is not identifiable, then it is not possible to uniquely identify its parameters from measured data. This contribution describes how solvers for differential-algebraic equations (DAE) can be used to examine if a model structure is locally identifiable. The procedure can be applied to both linear and nonlinear systems. If a model structure is not identifiable, it is also possible to examine which functions of the parameters that are locally identifiable.
\end{abstract}

Keywords: Identifiability, Nonlinear systems, Modelling, Identification, Descriptor systems

\section{INTRODUCTION}

Identifiability of a model structure means that it is possible to estimate unknown parameters uniquely from measured input and output data. One important reason to examine identifiability of a model structure is that the parameters represent physical properties that are of interest. Another reason is that numerical search methods might have difficulties estimating the parameters if the model structure is not identifiable.

Identifiability has been studied by many authors, e.g., (Walter, 1982). (Ljung, 1999) is a standard reference in system identification, including identifiability. In (Ljung and Glad, 1994), a general method for examining identifiability in linear and nonlinear systems, both state-space systems and differential-algebraic equations (DAE), using differential algebra is presented. The method pre-

\footnotetext{
1 This work has been supported by the Swedish Foundation for Strategic Research (SSF) through VISIMOD and EXCEL and by the Swedish Research Council (VR) which is gratefully acknowledged.
}

sented in this paper is inspired by the method in (Ljung and Glad, 1994), but does not use differential algebra. Instead solvers for differentialalgebraic equations are used to examine local identifiability. These solvers will be referred to as DAE solvers. DAE solvers have been developed to simulate models generated by objectoriented modeling languages such as Modelica (Tiller, 2001; Fritzson, 2004).

The basic principles behind the method presented in this paper are shown in the following example.

Example 1. Consider the model structure

$$
\ddot{y}(t)+2 \theta_{0} \dot{y}(t)+\theta_{0}^{2} y(t)=0
$$

which was examined in (Ljung and Glad, 1994). This model structure is globally identifiable, which was proved in (Ljung and Glad, 1994). Here it will be shown how a DAE solver can be used to prove local identifiability. If (1) is identifiable, it should be possible to compute the value of $\theta_{0}$ given measurements of $y$ generated from (1). This means that the following system of equations should be uniquely solvable for $\theta(t)$ : 


$$
\begin{aligned}
\ddot{y}(t)+2 \theta_{0} \dot{y}(t)+\theta_{0}^{2} y(t) & =0 \\
\ddot{y}(t)+2 \theta(t) \dot{y}(t)+\theta^{2}(t) y(t) & =0
\end{aligned}
$$

Simulating (2) using Dymola for $\theta_{0}=3, y(0)=0$, and $\dot{y}(0)=1$ gives the solution $\theta(t) \equiv 3$, so the model structure is locally identifiable at 3 . Other values of $\theta_{0}$ give corresponding results.

\section{BACKGROUND}

In this section, necessary background information on DAE solvers and identifiability is presented.

\subsection{DAE Solvers}

This section introduces the basic functionality of DAE solvers, and assumptions about the solver that will be needed to derive some of the results. The basic functionality that is assumed is that given a nonlinear DAE, $F(\dot{x}(t), x(t), t)=0$, the solvers produce $x(t)$ for a desired time interval.

Purely numeric solvers for DAE only handle a limited class of DAE, usually index 1 systems, or limited classes of higher index systems. The index of a DAE is basically the number of times the equations must be differentiated to solve $\dot{x}$ as a function of $x$ (e.g., Brenan et al., 1996). In this contribution it will not be sufficient to treat lower index problems, so instead the kind of solvers that are used to simulate object oriented models, such as Modelica models, will be used. Such solvers are included in, e.g., Dymola and OpenModelica. These solvers typically reduce the index to 1 by differentiating equations that are chosen using Pantelides' algorithm (Pantelides, 1988) and structure the equations so that large DAE systems can be simulated efficiently. Then a numerical solver is used. A detailed discussion on the construction of such solvers is out of the scope of this paper, but a discussion about them can be found in, e.g., (Fritzson, 2004).

During the index reduction process, some of the variables $x(t)$ are selected as states. For the user, this means that initial values of these variables can be selected independently of each other, while the initial values of the remaining variables are computed from the initial values of the states. It is possible for the user to influence the state selection process by indicating that some variables are preferred as states.

To derive the identifiability results some assumptions on the DAE solver are needed: (1) If locally unique solutions to the DAE exist, one of them is given. Otherwise the user is informed either that no solutions exist or that existing solutions are not locally unique. (2) Some of the variables that appear differentiated are selected as states by the solver. The initial values of these variables can be selected by the user, and the remaining initial values are computed from the states. (3) The number of unknowns must be the same as the number of equations. (The derivative of a variable does not count as an unknown.)

The assumptions represent a kind of ideal solver, but existing DAE solvers such as the one in Dymola come quite close to satisfying them.

\subsection{Identifiability}

Identifiability of a model structure basically means that if two parameter values $\theta_{1}$ and $\theta_{2}$ give the same predictor, then $\theta_{1}=\theta_{2}$. This property can be local or global. Noise models will not be treated in this contribution, so here the predictor is the simulated output. Noise models typically do not influence the identifiability of the actual system parameters. For a further discussion on this, see (Ljung and Glad, 1994) from which we also have taken the basic setting for this paper. This means that the model is specified by $r$ scalar equations,

$$
g_{i}(u, y, x, \dot{x}, \theta)=0 \quad i=1,2, \ldots, r .
$$

Here $u$ and $y$ are measured input and output signals, $x$ is a vector of internal variables, and $\theta$ is a vector of unknown parameters. The dimensions of $u, y, x$, and $\theta$ are $n_{u}, n_{y}, n_{x}$, and $n_{\theta}$. Also, $n_{\dot{x}}$ is the number of $x$ that appear differentiated. It is assumed that $r=n_{y}+n_{x}$ so that there are the same number of equations as unknowns if $u$ and $\theta$ are given. Furthermore it is assumed that the functions $g_{i}$ can be differentiated with respect to time as many times as necessary. Note that many linear and nonlinear model structures fit into this framework. Also note that higher derivatives of $x$ can be handled by extending the vector $x$.

In the system identification problem, it is also known that the parameters are constant, i.e.,

$$
\dot{\theta}=0 .
$$

The equation system (3) and (4) is generally not solvable for arbitrary signals $u$ and $y$ since there are more equations than unknowns. However, if the signals $u$ and $y$ come from an identical system, with some fixed parameter values $\theta_{0}$, the system (3) must have a solution with constant parameters, with $\theta \equiv \theta_{0}$. If the system is not identifiable, there will be more solutions than this one. The complete problem is thus to check if the following problem has a unique solution $\theta=\theta_{0}$ :

$$
\begin{aligned}
g_{i}\left(u, y, x, \dot{x}, \theta_{0}\right) & =0 & & i=1,2, \ldots, r \\
g_{i}(u, y, \tilde{x}, \dot{\tilde{x}}, \theta) & =0 & & i=1,2, \ldots, r \\
\dot{\theta} & =0 & &
\end{aligned}
$$

If there is only one solution, it must be $\theta \equiv \theta_{0}$ and the system is globally identifiable at $\theta_{0}$. If there 
are a number of distinct constant solutions $\theta$, the system is locally, but not globally identifiable at $\theta_{0}$. If there are an infinite number of solutions in every neighborhood of $\theta_{0}$, then the system is neither globally nor locally identifiable at $\theta_{0}$.

The identifiability properties of a model structure is normally influenced by whether the initial condition $x(0)$ is known or has to be estimated. The most common case in applications is perhaps that $x(0)$ is unknown, but it could be known for example if an experiment is started in an equilibrium.

\section{EXAMINING IDENTIFIABILITY}

The basic idea of the method proposed here is to solve the system of differential-algebraic equations (5) using a DAE solver to see if there is a locally unique solution $\theta \equiv \theta_{0}$. This section describes how the equations (5) should be preprocessed to allow them to be solved using a DAE solver, and how conclusions about identifiability can be drawn from the solution.

\subsection{Preprocessing}

Basically there are three problems with the description (5) when it comes to simulation with a DAE solver: (1) The number of equations are not necessarily the same as the number of unknowns. As was discussed in Section 2.1, DAE solvers cannot handle this situation. (2) Some of the $\theta$ may be selected as states by the solver so that initial conditions must be specified. This is a problem since the goal is to compute the value of $\theta$. (3) Some of the $\tilde{x}$ may be selected as states so that initial conditions must be specified. This is acceptable if $x(0)$ is known in the identification problem under examination, but otherwise this is undesirable.

Problem 2 is caused by the fact that derivatives of $\theta$ are included in the system of equations. To resolve problem 1 and 2, (5c) must be removed. At the same time new equations must be added by differentiating the given equations to make the number of unknowns the same as the number of equations. Note that the unknowns are $y, x, \tilde{x}$, and $\theta$ so the number of unknowns is initially $n_{y}+2 n_{x}+$ $n_{\theta}$, and that the number of equations (excluding $\dot{\theta}=0$ which should be removed) is $2 r=2 n_{y}+2 n_{x}$. If $n_{y}<n_{\theta}$ (which is usually the case), $n_{\theta}-n_{y}$ equations plus one equation for each new variable that is introduced in the process must be added.

The transformations may introduce new solutions that were not present in (5). However, the only equations that have been removed are $(5 \mathrm{c})$, so if the solution satisfies $\dot{\theta}=0$ it must be a solution of (5). This will be utilized later.
The case with unknown initial conditions $x(0)$ is more involved, so the cases with known and unknown initial conditions are discussed separately below.

3.1.1. Known Initial Conditions If the initial conditions are known, what needs to be done is to create $n_{\theta}-n_{y}$ new equations by differentiating equations from (5b) with respect to time. The equations that are differentiated should be chosen among those containing $\theta$ since the knowledge that $\dot{\theta}=0$ can be utilized here. Differentiating other equations actually does not introduce any new information to the DAE solver, since it can differentiate equations algebraically. It is preferable to differentiate several different equations containing elements of $\theta$ since higher derivatives may make the equations more difficult to handle for the DAE solver.

3.1.2. Unknown Initial Conditions If the initial conditions $x(0)$ are unknown, it is not acceptable if $\tilde{x}$ are selected as states by the DAE solver. To avoid this, $\dot{\tilde{x}}$ are not marked as derivatives of $\tilde{x}$ for the solver, but merely as time-dependent variables. To emphasize this, we will write $\tilde{x}_{p}$ instead of $\dot{\tilde{x}}$. This will introduce $n_{\dot{x}}$ new variables, so more equations need to be differentiated than for known initial conditions. Also, when differentiating, e.g., an equation containing $\tilde{x}_{p}$ this will produce the second derivative of $\tilde{x}$, which will also be a new variable, denoted $\tilde{x}_{p p}$. This means that more equations need to be differentiated in this case than for the case with known initial conditions.

When differentiating equations, one should select equations to be differentiated in systematic manner, so that not too many equations are differentiated. First, equations that do not introduce new variables (e.g., $\tilde{x}_{p p}$ ) should be differentiated. After that, groups of equations that when differentiated give more new equations than unknowns should be differentiated. In this process, equations containing $\theta$ should be selected first since the knowledge that $\dot{\theta}=0$ can be utilized here. Also note that all derivatives of $y$ should be considered as known since the DAE solver can compute them from (5a). This process will eventually give the same number of equations as unknowns.

The procedure outlined above can be formalized using minimally structurally singular (MSS) sets (Pantelides, 1988). A set of equations is structurally singular with respect to a set of variables if the number of equations are greater than the number of variables, and a set of equations is minimally structurally singular if it is structurally singular, and none of its proper subsets are structurally singular. The following property of MSS sets will be needed: 
Lemma 2. If a set of equations is MSS with respect to the variables occurring in the equations, then the number of equations is exactly one more than the number of variables.

Proof. The number of equations must be greater than the number of variables, otherwise the set of equations would not be structurally singular. If the number of equations exceeds the number of variables by two or more, it would be possible to remove one equation and still have a set of equations that is structurally singular. Therefore, the number of equations must exceed the number of variables by exactly one.

The following algorithm for differentiating equations can now be formulated:

(1) Let $E$ be the original set of $n_{x}+n_{y}$ equations in $(5 \mathrm{~b})$.

(2) Let $z$ be a set with $n_{x}$ elements, that for each element in $\tilde{x}$ contains the highest derivative that occurs in $E$.

(3) Find a set of equations from $E$ that is MSS with respect to the variables in $z$ occurring in the set. This can be done, e.g., using Algorithm 3.2 in (Pantelides, 1988). Preferably, the MSS set should have as few equations as possible.

(4) Differentiate the equations in the MSS set. According to Lemma 2, the number of new equations generated will exceed the number of new variables generated by one.

(5) In $E$, replace the equations in the MSS set with their differentiated versions.

(6) Repeat from 2 until the number of equations, including those in $E$ and those that have been removed from $E$, equals the number of unknowns, that is the number of $x$ including differentiated versions plus the number of $\theta$.

The algorithm will terminate since the difference between the number of equations and number of unknowns is reduced by one for each run.

\subsection{Drawing Conclusions on Identifiability}

After the preprocessing step, there are as many equations as unknowns, so the transformed equations can be plugged into a DAE solver. What should be done now is thus to simulate the transformed equations, and examine if there is a unique solution with $\theta \equiv \theta_{0}$. Before simulating, the input signal $u$, initial condition $x(0)$ and value of $\theta_{0}$ should be selected to the values where identifiability should be checked. Here it can be noted that identifiability properties often are the same for most inputs, initial states and $\theta_{0}$, see e.g., (Ljung and Glad, 1994). Furthermore, the most time con- suming parts of the process are the preprocessing and index reduction step in the solver, and these do not have to be repeated when changing $u$, $x(0)$, and $\theta_{0}$. This means that several different choices can be tested with small computational effort. After making these choices and running the DAE solver, there are basically five situations that may occur which lead to different conclusions on the identifiability. These situations are discussed below.

The Solution $\theta$ is Constant, and $\theta \equiv \theta_{0}$ : The only equation that was removed from the original set of equations is (5c). This equation is still fulfilled since $\theta$ is constant, so the present solution is a solution of the original equations (5). Furthermore, since the DAE solver is assumed to give an error message if a solution is not locally unique, the solution $\theta \equiv \theta_{0}$ is locally unique. This gives that the system is locally identifiable at $\theta_{0}$.

The Solution $\theta$ is Constant, and $\theta \not \equiv \theta_{0}$ : As in the case where $\theta \equiv \theta_{0}$, it is clear that the present solution is a solution of the original equations (5) as $\theta$ is constant. Since $\theta \not \equiv \theta_{0}$ it is proved that there are two different values of $\theta$ that give the same input-output behavior, so the model is not globally identifiable at $\theta_{0}$. However, it is locally identifiable at the constant value $\theta$ since this solution is locally unique according to the assumptions on the DAE solver.

If it is desirable to determine if the model is locally identifiable also at $\theta_{0}$, one should go back and run the simulation with a different choice of $u$ and/or $x(0)$ to see if the new solution has $\theta \equiv \theta_{0}$.

If the functions $g_{i}$ are polynomial, the results in (Ljung and Glad, 1994) give that a model structure is either locally identifiable for all $\theta$ or for no $\theta$. If the $g_{i}$ are polynomial, it is therefore clear that the model structure is locally identifiable also at $\theta_{0}$.

The Solution $\theta$ is Time-Varying: The original set of equations included $\dot{\theta}=0$, so any timevarying solutions must have been introduced by the preprocessing step. One remedy to this problem is to try different $u$ and $x(0)$ to see if this gives constant $\theta$. Otherwise, it is necessary to return to the preprocessing step and differentiate a different set of equations.

The DAE Solver Indicates that Existing Solutions are not Locally Unique: If existing solutions are not locally unique, the preprocessing step has either introduced new solutions, or the model structure is not locally identifiable at $\theta_{0}$. The case with solutions that have been added in the 
preprocessing step can be handled by returning to the preprocessing, differentiating a different set of equations and then running the simulation again. If the cause of a non-unique solution is that the model structure is not locally identifiable at $\theta_{0}$, then this can be verified by computing which parameters or functions of the parameters that are identifiable. This is discussed in Section 3.3.

The DAE Solver Indicates that no Solutions Exist: This is a degenerate case since it is clear that at least one solution exists: $\tilde{x}=x$ and $\theta=\theta_{0}$. The reason is usually that the initial condition $x(0)$ gives problems for the solver. For example, $x(0)=0$ may give rise to problems. If this problem occurs, run the simulation again with a new selection of $x(0)$ and/or $u$.

The discussion above can be summarized with the following theorem.

Theorem 3. Assume that if a locally unique solution to (5) exists, then it is given by the DAE solver. Otherwise the user is notified that no solution exists or that existing solutions are not locally unique. Then, for the five situations that can occur, the following conclusions can be made about identifiability for the selected input $u$ and initial state $x(0)$ :

(1) If the solution $\theta$ is constant, and $\theta \equiv \theta_{0}$, then the model structure is locally identifiable at $\theta_{0}$.

(2) If the solution $\theta$ is constant, and $\theta \not \equiv \theta_{0}$, then the model structure is locally identifiable at $\theta$ and not globally identifiable at $\theta_{0}$.

(3) If the solution $\theta$ is time-varying, then the current solution has been introduced by the preprocessing step.

(4) If the DAE solver indicates that existing solutions are not locally unique, then the model structure is either not locally identifiable at $\theta_{0}$ or new solutions have been introduced by the preprocessing step.

(5) If the DAE solver indicates that no solutions exist, numerical problems have occurred.

\subsection{Identifiable Functions of Parameters}

If a model structure is not locally identifiable, it may be interesting to examine if some parameters or functions of parameters are identifiable. This makes it possible to prove that a model structure is not locally identifiable at a certain parameter value. It may also be interesting to know which parameters that are identifiable if they represent physical quantities.

The basic observation that is used to find identifiable functions of parameters is that if a model structure is not identifiable, then it should be possible to make it identifiable by fixing one or more parameters. If for example $a+b$ is identifiable but not $a$ and $b$, then $b$ can be made identifiable by fixing $a$ to, e.g., $a=1$. As it is not possible to know beforehand which parameters that can be fixed, all parameters and combinations of parameters have to be tried until the model structure becomes identifiable. For each parameter (each element of $\theta)$ it is tested if the model becomes identifiable with this parameter fixed to the value of the corresponding element in $\theta_{0}$. This is checked by restarting the procedure from the preprocessing step. If cannot be made identifiable by fixing one parameter, then combinations of two parameters are tested, then combinations of three parameters and so on.

When a parameter, or combination of parameters, that when fixed make the model structure identifiable has been found, it is still necessary to show that the model actually is not identifiable. This is because the extra solutions that were reported by the DAE solver may be a result of the preprocessing step. To prove this, the value of the parameters are changed from their corresponding values in $\theta_{0}$. If the simulation procedure still gives constant values for all parameters, it has been proven that the model structure is not globally identifiable since there are more than one set of parameter values that give the same input-output behavior. Local identifiability can be tested by making small changes in the parameters.

When some parameters that can be fixed have been found, it may be interesting to examine how the values of these parameters affect the value of the other parameters. This can be done by varying the fixed parameters to different fixed values, and noting the values of the other parameters. In this way it is possible to determine which function of the parameters that is identifiable.

\section{EXAMPLE}

In this section, the identifiability checking procedure is exemplified on a compartmental model.

Example 4. In this example the following model structure from (Ljung and Glad, 1994) is studied:

$$
\begin{aligned}
\dot{x}(t) & =-\frac{V_{m} x(t)}{k_{m}+x(t)}-k_{01} x(t) \\
x(0) & =D \\
y(t) & =c x(t)
\end{aligned}
$$

Let the initial condition $D$ be known, so that the unknown parameters are

$$
\theta=\left(\begin{array}{llll}
V_{m} & k_{m} & k_{01} & c
\end{array}\right)^{T} .
$$


Assume that identifiability is to be tested at $\theta_{0}=$ $\left(\begin{array}{llll}1 & 2 & 3 & 4\end{array}\right)^{T}$. The basic setup to be simulated (5) is then

$$
\begin{aligned}
\dot{x}(t) & =-\frac{1 \cdot x(t)}{2+x(t)}-3 \cdot x(t) \\
y(t) & =4 \cdot x(t) \\
\dot{\tilde{x}}(t) & =-\frac{V_{m}(t) \tilde{x}(t)}{k_{m}(t)+\tilde{x}(t)}-k_{01}(t) \tilde{x}(t) \\
y(t) & =c(t) \cdot \tilde{x}(t) \\
\theta(t) & =\left(V_{m}(t) k_{m}(t) k_{01}(t) c(t)\right)^{T} \\
\dot{\theta}(t) & =0
\end{aligned}
$$

In the preprocessing step, (8f) should be removed, and $n_{\theta}-n_{y}=3$ new equations should be added by differentiating equations. Here, $(8 \mathrm{c})$ is differentiated twice and (8d) is differentiated once to get three new equations. ( $8 \mathrm{c}$ ) is chosen to be differentiated twice since it contains several parameters. The initial value is set to $x(0)=\tilde{x}(0)=1$. Simulating the new system using the DAE solver in Dymola gives $\theta(t) \equiv\left(\begin{array}{llll}1 & 2 & 3 & 4\end{array}\right)^{T}$, so the model structure is locally identifiable at this parameter value.

On the contrary to what was done above, assume now that identifiability should be examined for the case when the initial condition $x(0)$ is unknown. In this special case it is possible to see that the model structure is not identifiable without going through the preprocessing step again. This is done by simulating the same system as above but with $x(0) \neq \tilde{x}(0)$. Doing this gives a constant $\theta(t)$, but with $\theta(t) \neq \theta_{0}$. This directly shows that the model structure is not identifiable with unknown initial conditions. To examine which functions of the parameters that are identifiable, several different values of $\tilde{x}(0)$ are tried, and for each case the value of $\theta(t)$ is noted. During this procedure $k_{01}(t)$ is always at its true value, $k_{01}(t)=3$, so this parameter is identifiable. The other parameters vary when $\tilde{x}(0)$ is varied, so they are not identifiable. To illustrate which functions of the parameters that are identifiable, $k_{m}$ and $c$ are plotted against $V_{m}$ in Fig. 1. The figure suggests that $V_{m} / k_{m}$ and $V_{m} \cdot c$ are identifiable. $\diamond$

\section{CONCLUSIONS}

We have studied how DAE solvers can be used to examine identifiability in a rather wide class of model structures. The main advantages of the method is that it can be used on many model structures, both linear and non-linear.

The preprocessing step, as it is described in this paper, is heuristic and may require manual intervention. Further research efforts could thus be put at making this process fully automatic. Such

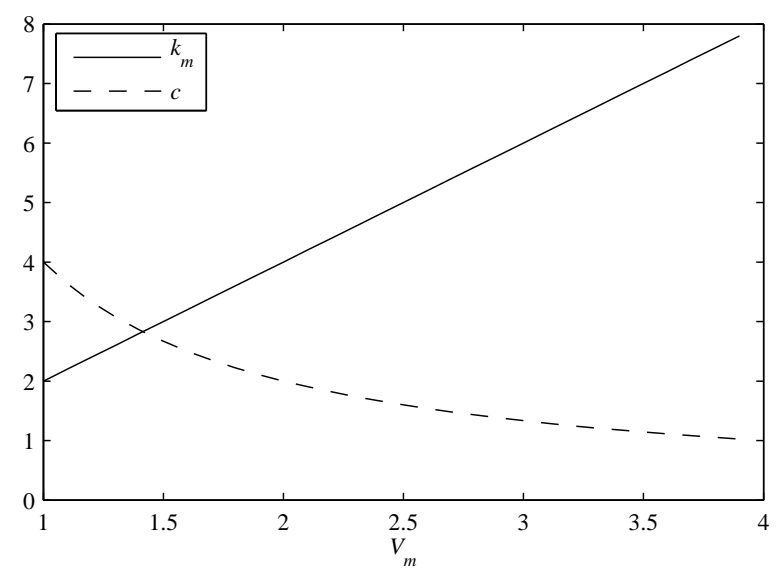

Fig. 1. Identifiable functions of parameters.

efforts should also be directed at examining larger examples than have been done in this paper.

An interesting aspect of the method in this paper is that DAE solvers make it possible to draw certain conclusions about dynamic systems. This could be applied to other areas of system analysis such as observability.

\section{REFERENCES}

Brenan, K. E., S. L. Campbell and L. R. Petzold (1996). Numerical Solution of Initial-Value Problems in Differential-Algebraic Equations. Classics In Applied Mathematics. SIAM. Philadelphia.

Fritzson, P. (2004). Principles of Object-Oriented Modeling and Simulation with Modelica 2.1. Wiley-IEEE. New York.

Ljung, L. (1999). System Identification - Theory for the User. Information and System Sciences Series. Second ed. Prentice Hall PTR. Upper Saddle River, N.J.

Ljung, L. and T. Glad (1994). On global identifiability for arbitrary model parametrizations. Automatica 30(2), 265-276.

Pantelides, C. C. (1988). The consistent initialization of differential-algebraic systems. SIAM Journal on Scientific and Statistical Computing 9(2), 213-231.

Tiller, M. (2001). Introduction to Physical Modeling with Modelica. Kluwer. Boston, Mass.

Walter, E. (1982). Identifiability of State Space Models with Applications to Transformation Systems. Vol. 46 of Lecture Notes in Biomathematics. Springer-Verlag. Berlin, Heidelberg, New York. 


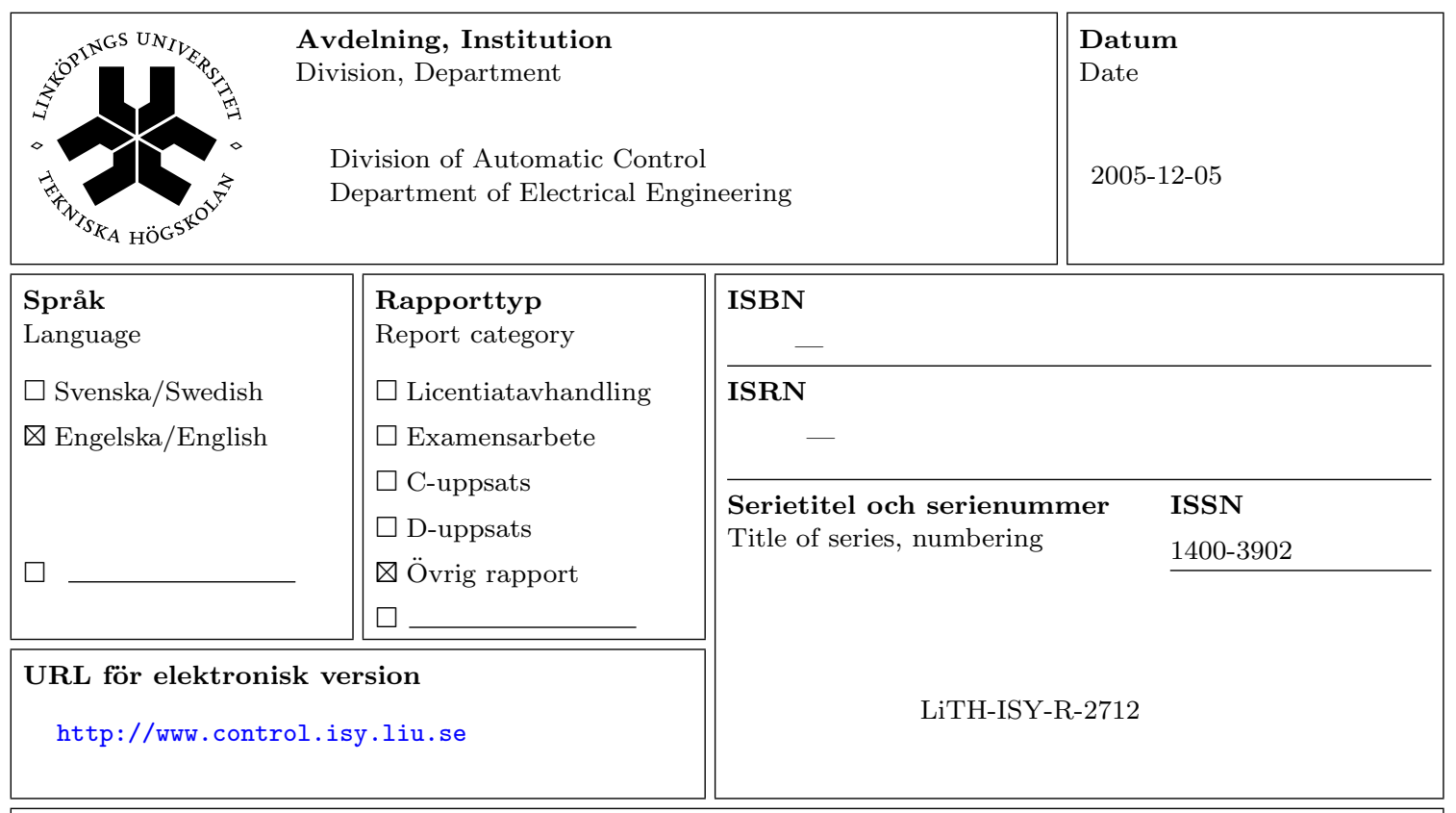

Titel Using DAE Solvers to Examine Local Identifiability for Linear and Nonlinear Systems

Title

Författare Markus Gerdin

Author

\section{Sammanfattning}

Abstract

If a model structure is not identifiable, then it is not possible to uniquely identify its parameters from measured data. This contribution describes how solvers for differential-algebraic equations (DAE) can be used to examine if a model structure is locally identifiable. The procedure can be applied to both linear and nonlinear systems. If a model structure is not identifiable, it is also possible to examine which functions of the parameters that are locally identifiable. 